\title{
Manuscript and Record Sources
}

\author{
NATIONAL REPOSITORIES
}

The National Archives: The Public Record Office

(Unless otherwise specified, all unpublished sources cited in this book are housed in The National Archives.)

Admiralty

ADM $75 \quad$ Royal Greenwich Hospital: Deeds

Chancery

$\mathrm{C}_{1}$

$\mathrm{C} 44$

$\mathrm{C} 47$

C 49

C 54

C 66

C 67

$\mathrm{C} 76$

C 81

C $133-9$

C 143

C 145

C 146

C 241

C 255

C 260

Six Clerks Office: Early Proceedings

Common Law Pleadings, Tower Series

Miscellanea

Parliamentary and Council Proceedings

Close Rolls

Patent Rolls

Supplementary Patent Rolls

Treaty Rolls

Warrants for the Great Seal, Series I

Inquisitions Post Mortem

Inquisitions Ad Quod Damnum

Miscellaneous Inquisitions

Ancient Deeds, Series C

Certificates of Statute Merchant and Statute Staple

Miscellaneous Files and Writs

Recorda

Common Pleas

CP 25/1

Feet of Fines

$\mathrm{CP} 40$

Plea Rolls

CP 52

Brevia Files 
Duchy of Lancaster
DL 27
Deeds, Series LS
DL 29
Accounts of Auditors, etc.

\section{Exchequer}

E 32

Justices of the Forest

E 36

Treasury of the Receipt: Miscellaneous Books

E 40

TR: Ancient Deeds, Series A

E 42

TR: Ancient Deeds, Series AS

E 43

TR: Ancient Deeds, Series WS

E 101

King's Remembrancer: Accounts Various

E 122

KR: Customs Accounts

E 142

KR: Extents, etc., of Forfeited Lands

E 149

KR: Escheators' Files

E 159

KR: Memoranda Rolls

E 179

KR: Subsidy Rolls

E 198

KR: Feudal Tenure and Distraint of Knighthood

E 199

KR and Lord Treasurer's Remembrancer: Sheriffs' Accounts, etc.

E 202

KR: Writs, Original Series

E 210

KR: Ancient Deeds, Series D

E 326

Augmentation Office: Ancient Deeds, Series B

E 329

AO: Ancient Deeds, Series BS

E 358

Pipe Office: Miscellaneous Enrolled Accounts

E 359

PO: Account Rolls of Subsidies and Aids

E 368

LTR: Memoranda Rolls

E 372

PO: Pipe Rolls

Itinerant Justices

JUST 1

Eyre Rolls, etc.

JUST 3

Gaol Delivery Rolls

King's Bench

$\mathrm{KB} 9$

Indictments Files, etc.

KB 27

Coram Rege Rolls

Palatinate of Durham

DURH 3

DURH 13

DURH 20
Chancery Court: Cursitor's Records

Assizes and Court of Pleas: Plea and Gaol Delivery

Rolls

Exchequer: Auditor's Records 
Special Collections

$\begin{array}{ll}\text { SC } 1 & \text { Ancient Correspondence } \\ \text { SC } 6 & \text { Ministers' and Receivers' Accounts } \\ \text { SC } 8 & \text { Ancient Petitions }\end{array}$

Other Sources

IND

Indexes to Various Series

PRO Domestic Records of the Public Record Office (including transcripts)

\section{British Library}

Additional Charters

Cotton Roll XIII.8 Earl of Northumberland's Retinue-list, 1385

Egerton Charters

Harley Charters

MSS Additional

$\begin{array}{ll}8835 & \text { Wardrobe Book, 1303-4 } \\ 28024 & \text { Beauchamp Cartulary, xv cent. } \\ \text { MSS Cotton } & \\ \text { Claudius D.IV } & \text { Durham Priory: Historical Collections, xv cent. } \\ \text { Faustina A.VI } & \text { Durham Priory Register, xiv cent. } \\ \text { Nero C.VIII } & \text { Wardrobe Accounts, 1310-37 } \\ \text { Tiberius E.VI } & \text { St Albans Abbey Register, xiv-xv cent. } \\ \text { Vitellius A.XX } & \text { Historical Collections, xiii cent. } \\ \text { MS Harley 4843 } & \text { Durham Priory: Historical Collections, xvi cent. } \\ \text { MSS Lansdowne } & \\ 397 & \begin{array}{l}\text { Durham Priory: Miscellaneous Collections, xiv } \\ \text { cent. }\end{array} \\ 902 & \text { Transcripts, xviii cent. } \\ \text { MSS Stowe } & \text { Wardrobe Book, 1322-3 } \\ 553 & \text { Durham Priory Register, xiii-xiv cent. } \\ 930 & \end{array}$

OTHER REPOSITORIES

\section{Alnwick Castle, Northumberland}

MS D.XI.1

Tynemouth Priory Cartulary, xiv cent.

Northumberland Collections Transcripts, xix cent. 


\section{Balliol College, Oxford}

E.4

Stamfordham and Heugh

\section{Bodleian Library, Oxford}

MSS Dodsworth 45, 78, 94, 149 Transcripts, xvii cent.

MS Eng. c. 7032

Towneley Papers, xvii cent.

MS Laud. Misc. 748

Durham Priory: Historical Collections, xv cent.

MS Tanner 197

Wardrobe Accounts, 1311

MS Top. Yorks. e. 8

Transcripts, xviii cent.

\section{Borthwick Institute, York}

Reg.9A-B Register of Archbishop William Melton (1317-40)

Reg.10

Reg.11 Register of Archbishop William Zouche (1342-52)

Reg.12 Register of Archbishop John Thoresby (1352-73) Register of Archbishop Alexander Neville (1374-88)

\section{Castle Howard, Yorkshire}

A1 Dacre and Greystoke

\section{Cockermouth Castle, Cumbria}

D/Lec/301 Lucy Cartulary, xvi cent.

\section{Cumbria Record Office}
(a) Carlisle
$\mathrm{D} / \mathrm{Ay}$
Aglionby
$\mathrm{D} / \mathrm{HA}$
Hough, Halton and Soal of Carlisle
$\mathrm{D} / \mathrm{HC}$ Howard of Corby
D/HGB
Blencow of Blencowe
D/Lons/L Lowther of Lowther, Earls of Lonsdale
D/MBS
$\mathrm{DMH}$ Mounsey, Bowman and Sutcliffe of Carlisle
D/Mus Mounsey-Heysham
D/Wal Musgrave of Edenhall Walton of Alston 
D/Wyb Wybergh

MS Machell 5 Transcripts, xvii cent.

(b) Kendal

WD/Crk Crackanthorpe of Newbiggin

WD/Ry Le Fleming of Rydal Hall

\section{Durham Cathedral Library}

MS Raine $52 \quad$ Transcripts, xix cent.

MSS Randall 3, 5 Transcripts, xviii cent.

\section{Durham County Record Office, Durham}
$\mathrm{D} / \mathrm{Gr}$
Greenwell Deeds
$\mathrm{D} / \mathrm{Lo}$
Londonderry Estates
$\mathrm{D} / \mathrm{Sa}$
Salvin of Croxdale
D/Sh.H
Sherburn Hospital
$\mathrm{D} / \mathrm{St}$
Strathmore Estate

Durham University Library, Archives and Special Collections

(a) Palace Green

HNP/N Howard Family: Northumberland

SGD 54 Littleburn, Holywell and Nafferton

(b) 5 The College

Durham Cathedral Muniments

Original Deeds, etc.

Elemos.

Elemosinaria

Finc.

Finchalia

Haswell Deeds

Loc.

Locelli

Misc. Ch.

Miscellaneous Charters

Pont.

Pontificalia

Reg.

Regalia

Sacr.

Sacristaria

SHD

Sherburn Hospital

Spec.

Specialia 
Other Sources

Bursar's Accounts

Cart. I-IV

Cartularia, $\mathrm{xv}$ cent.

Cart. Vet.

Cartuarium Vetus, xiii cent.

Reg. II

Priory Register, xiv-xv cent.

Reg. Hatfield

Register of Bishop Thomas Hatfield (1345-81)

Rep. Mag.

Repertorium Magnum, xv cent.

Essex Record Office, Chelmsford

D/DBy/T27 Miscellaneous Deeds

Guildhall Library, London

MS 31302 Skinners' Company

John Rylands Library, Deansgate, Manchester

Latin MS 236 Accounts of Queen's Household, 1357-8

$\mathrm{PHC}$

Phillipps Charters

Lancashire Record Office, Preston

DDTO Towneley of Towneley

\section{Levens Hall, Cumbria}

Medieval Deeds

Lincolnshire Archives, Lincoln

Dean and Chapter Muniments

Dij/62/iii Lincolnshire Churches

Merton College, Oxford

Stillington Deeds 
Northamptonshire Record Office, Northampton

Stopford-Sackville Muniments

\section{Northumberland Collections Service, Woodhorn}

324

Blackett-Ord of Whitfield

SANT/TRA Society of Antiquaries of Newcastle upon Tyne:

Transcripts of Records

Waterford Charters

ZBL

Blackett of Matfen

ZMI

Middleton of Belsay

ZSW

Swinburne of Capheaton

North Yorkshire County Record Office, Northallerton

ZAZ

$\mathrm{ZBO}$

ZIQ

$\mathrm{ZQH}$

$\mathrm{DD} / 4 \mathrm{P}, 6 \mathrm{P}$

$\mathrm{DD} / \mathrm{FJ}$
Hutton of Marske

Bolton Hall

Meynell of Kilvington

Chaytor of Croft

\section{Nottinghamshire Archives, Nottingham}

Nottingham University, Manuscripts and Special Collections

$\mathrm{PL} / \mathrm{E} 11$

Dukes of Portland: Northumberland Estates

\section{St George's Chapel Archives and Chapter Library,}

Windsor Castle

XI.K. Ancient Deeds

Shakespeare Birthplace Trust, Stratford-upon-Avon

DR 10

Gregory-Hood of Stivichall 
MANUSCRIPT AND RECORD SOURCES

Society of Antiquaries, London

MS 120

Wardrobe Book, 1316-17

MS 121

Wardrobe Book, 1317-18

York Minster Library and Archives

MS XVI.A.1 Cartulary of St Mary's Abbey, York, xiv cent. 